\title{
Estimation of optical parameters of very thin films
}

\author{
Ernesto G. Birgin * Ivan E. Chambouleyron ${ }^{\dagger} \quad$ José Mario Martínez ${ }^{\ddagger}$ \\ Sergio D. Ventura $\S$
}

April 18, 2002

\begin{abstract}
In recent papers, the problem of estimating the thickness and the optical constants (refractive index and absorption coefficient) of thin films using only transmittance data has been addressed by means of optimization techniques. Models were proposed for solving this problem using linearly constrained optimization and unconstrained optimization. However, the optical parameters of "very thin" films could not be recovered with methods that are successful in other situations. Here we introduce an optimization technique that seems to be efficient for recovering the parameters of very thin films.
\end{abstract}

Keywords: Optical constants, thin films, optimization, numerical algorithms.

\section{Introduction}

We consider the problem of estimating the refractive index, the absorption coefficient and the thickness of a thin film, using transmittance data. Given the wavelength $\lambda$, the refractive index of the substrate $s$ and the unknowns $d$ (thickness), $n(\lambda)$ (refractive index) and $\alpha(\lambda)$ (absorption coefficient), the theoretical transmittance is given by well-known formulae $[1,2,3,8]$. Having the transmittance data for many different wavelengths, we want to estimate the above mentioned unknowns. At a first glance, this problem is highly underdetermined since, for each wavelength, the single equation

Theoretical Transmittance $=$ Measured Transmittance

*Department of Computer Sciences IME-USP, University of São Paulo, Rua do Matão 1010, Cidade Universitária, 05508-090, São Paulo SP, Brazil. This author was supported by PRONEX-Optimization 66.2199/1997-1, FAPESP (Grants 99/08029-9 and 01/04597-4) and CNPq (Grant 300151/00-4). e-mail: egbirgin@ime.usp.br

${ }^{\dagger}$ Department of Applied Physics, Institute of Physics, University of Campinas, CP 6065, 13083-970 Campinas SP, Brazil. This author was supported by FAPESP and CNPq. e-mail: ivanch@ifi.unicamp.br

${ }^{\ddagger}$ Department of Applied Mathematics IMECC-UNICAMP, University of Campinas, CP 6065, 13081-970 Campinas SP, Brazil. This author was supported by PRONEX-Optimization 66.2199/1997-1, FAPESP (Grant 01/04597-4), CNPq and FAEP-UNICAMP. e-mail: martinez@ime.unicamp.br

${ }^{\S}$ Department of Applied Mathematics IMECC-UNICAMP, University of Campinas, CP 6065, 13081-970 Campinas SP, Brazil. e-mail: ventura@ime.unicamp.br 
has three unknowns $d, n(\lambda), \alpha(\lambda)$ and only $d$ is repeated for all values of $\lambda$. The underdetermination can be circumvented incorporating prior knowledge on the functions $n(\lambda)$, $\alpha(\lambda)$ in order to decrease the degrees of freedom of (1) at a point that only physically meaningful estimated parameters are admitted.

The idea of assuming a closed formula for $n$ and $\alpha$ depending on few coefficients has been exploited in celebrated methods [8]. The methods originated on this idea are efficient when the transmittance curve exhibits some fringe patterns, representing rather large zones of the spectrum were $\alpha(\lambda)$ is almost null. In other cases, the fulfillment of (1) can be very crude or the curves $n(\lambda), \alpha(\lambda)$ are physically unacceptable.

In $[2,3]$, instead of imposing a functional form for $n(\lambda)$ and $\alpha(\lambda)$, the phenomenological constraints that restrict the variability of these functions were stated explicitly so that the estimation problem took the form

$$
\text { Minimize } \sum_{\lambda}[\text { Theoretical Transmittance }(\lambda)-\text { Measured Transmittance }(\lambda)]^{2}
$$

$$
\text { subject to Physical Constraints. }
$$

In this way, well behaved functions $n(\lambda)$ and $\alpha(\lambda)$ can be obtained without severe restrictions that could damage the quality of the fitting (1). In [1] the physical constraints were handled in such a way that the final problem turned out to be unconstrained and could be solved by means of an efficient large-scale minimization method (see [7]).

The method introduced in [1], called PUMA (pointwise unconstrained minimization approach) from now on, was able to solve both computer-generated ("gedanken") and real problems of film parameter estimation (see $[1,6]$ ) but failed in the case of very thin films (of thickness, say, smaller than $80 \mathrm{~nm}$ ). This motivated us to seek algorithms that could handle thinner films in an efficient way.

Let us describe now the main ideas of the algorithm. As was mentioned in $[1,2]$, the problem (2-3) has infinitely many solutions because the system

$$
\begin{gathered}
\text { Theoretical Transmittance }(\lambda)=\text { Measured Transmittance }(\lambda) \forall \lambda \\
\text { and Physical Constraints }
\end{gathered}
$$

is, in general, underdetermined. The solutions are the possible values of thickness and the possible refraction and absorption functions that satisfy (4-5). However, when the films are not very thin, the information contained in (4-5) is enough to guarantee that all the solutions are reasonably close to the true parameters. This is not the case when the films are extremely thin. In the extreme case, when the thickness is zero, the true refraction and absorption coefficients do not influence in the transmittance at all. Therefore, transmittance data cannot give information about those parameters, no matter the cleaverness of the optimization algorithm. This means that, when the film is very thin, some additional information must be added to (3) in order to reduce the degrees of freedom of the system. The most obvious way to do this is to impose functional forms to the index of refraction and to the absorption. However, although a functional form for the refractive index, as a function of the wavelength, is generally accepted, this is not the case for the absorption coefficient. For the type of films in which we are interested, we only know that the logarithm of $\alpha$ (the absorption coefficient) as a function of the photon energy, has a 
functional form that resembles the integral $\left(\int\right)$ mathematical symbol. On the other hand, a suitable functional form must have a sufficiently large number of parameters in order to cover a sufficient amount of possibilities. In other words, choosing the model involves a careful decision combining degrees of freedom and a priori information.

This paper is organized as follows. Section 2 introduces the mathematical model for the parameters estimation problem. In Section 3 the optimization procedure is described. Numerical results on gedanken and real films are shown in Section 4. Some conclusions are drawn in Section 5.

\section{The model}

We consider a (thin) film deposited on a (thick) transparent substrate. The theoretical formulae that give the measured transmitted energy as a function of the wavelength $\lambda$ (see, for example, [8]) are:

$$
T=\text { Transmittance }=\frac{A x}{B-C x+D x^{2}}
$$

where

$$
\begin{gathered}
A=16 s\left(n^{2}+\kappa^{2}\right) \\
B=\left[(n+1)^{2}+\kappa^{2}\right]\left[(n+1)\left(n+s^{2}\right)+\kappa^{2}\right] \\
C=\left[\left(n^{2}-1+\kappa^{2}\right)\left(n^{2}-s^{2}+\kappa^{2}\right)-2 \kappa^{2}\left(s^{2}+1\right)\right] 2 \cos \varphi \\
-\kappa\left[2\left(n^{2}-s^{2}+\kappa^{2}\right)+\left(s^{2}+1\right)\left(n^{2}-1+\kappa^{2}\right)\right] 2 \sin \varphi \\
D=\left[(n-1)^{2}+\kappa^{2}\right]\left[(n-1)\left(n-s^{2}\right)+\kappa^{2}\right] \\
\varphi=4 \pi n d / \lambda, \quad x=\exp (-\alpha d), \quad \alpha=4 \pi \kappa / \lambda .
\end{gathered}
$$

For future use, we also define the photon energy $E(\mathrm{eV})=1240 / \lambda(\mathrm{nm})$. In $(6-11), d$ is the thickness of the film, $s$ is the refractive index of the substrate, $n$ is the refractive index of the film, $\alpha$ is the absorption coefficient and $\kappa$ is the (adimensional) attenuation coefficient.

Our objective is to estimate $d, n=n(\lambda)$ and $\alpha=\alpha(\lambda)$, given a table of measured transmittances $\left(\lambda_{i}, T_{i}^{\text {meas }}\right), i=1, \ldots, M$. We propose a model where:

(a) The quantity $1 /\left[n(\lambda)^{2}-1\right]$ is a linear function of $1 / \lambda^{2}$ (see $[9]$ ).

(b) The display of the function $\log [\alpha(\lambda)]$ exhibits an "integral shape" $\left(\int\right)$ when expressed as a function of the photon energy $E$. In other words, the function $\log [\bar{\alpha}(E)] \equiv$ $\log [\alpha(1240 / \lambda)]$ has the form specified above.

The fact described in (b) lead us to investigate families of functions where the integral shape is present. Let $\mathcal{F}(\gamma, \eta)$ the set of twice continuously differentiable functions $\psi$ : $\mathbb{R} \rightarrow \mathbb{R}$ such that:

(i) $\psi(0)=\gamma$;

(ii) $\psi^{\prime}(0)=\eta$;

(iii) $\psi^{\prime \prime}(0)=0$; 
(iv) $\psi^{\prime}(t) \geq 0$ for all $t \in \mathbb{R}$.

(v) $\psi^{\prime \prime}(t)>0$ if $t<0$ and $\psi^{\prime \prime}(t)<0$ if $t>0$.

It is easy to see that, given $\psi_{-}, \psi_{+} \in \mathcal{F}(\gamma, \eta)$, the function $\psi$ defined by

$$
\psi(t)=\psi_{-}(t) \text { if } t \leq 0
$$

and

$$
\psi(t)=\psi_{+}(t) \text { if } t \geq 0,
$$

also belongs to $\mathcal{F}(\gamma, \eta)$. This property allows to define functions that satisfy the properties (i)-(v) and are not odd. We selected four functions $\theta_{j} \in \mathcal{F}\left(\gamma_{j}, \eta_{j}\right), j=1,2,3,4$, choosing $\gamma_{j}$ and $\eta_{j}$ in such a way that $\theta_{j}\left(\left[E_{\min }, E_{\max }\right]\right) \subset[0,1]$, where $E_{\min }$ and $E_{\max }$ are the minimum and the maximum photon energy in the spectrum under consideration, corresponding, respectively, to the maximum and minimum wavelength. Finally, we propose the following form for the logarithm of the absorption coefficient:

$$
\operatorname{LogModel}-\alpha(E)=\sum_{i=1}^{4} a_{i} \theta_{i}\left[b_{i}(E-c)\right]+k .
$$

As we mentioned above [9], it is generally accepted that a suitable model of the refractive index comes from

$$
\frac{1}{n^{2}-1}=\frac{m}{\lambda^{2}}+\beta
$$

which yields

$$
\operatorname{Model-n}(\lambda)=\sqrt{\frac{1}{\frac{m}{\lambda^{2}}+\beta}+1} .
$$

We can define new variables, depending on $m$ and $\beta$, so that they are computationally more insightful. Indeed, once $m$ and $\beta$ are fixed, we define $p$ and $q$ through $p=n_{m, \beta}\left(\lambda_{\max }\right)$ and $q=n_{m, \beta}\left(\lambda_{\min }\right)-n_{m, \beta}\left(\lambda_{\max }\right)$ (the inverse relation being quite simple). From this definition it is clear that $p>1$ and $q>0$.

By noting that $n_{m, \beta}(\lambda)$ is a decreasing function of $\lambda$, we see that $p$ is the lowest function value while $q$ is its amplitude. For the absorption coefficient, we observe that $c$ is the common inflection point of the four functions being added up, while $k$ is the height of the resulting function. The four paired parameters $a_{j}, b_{j}$ control, for each function $\theta_{j}$, the vertical and horizontal functional stretching or shrinking. Observe that in this way, we must use $b_{i}, a_{i}>0$ in order to keep the integral shape of the resulting function. The functions $\theta_{i}$ used in our model were the following:

$$
\begin{aligned}
& \theta_{1}=\left\{\begin{array}{l}
\frac{2}{4+\pi}\left(\arctan (\bar{E})+\frac{\pi}{2}\right) \text { when } \bar{E}<0, \\
\frac{2}{4+\pi}(\operatorname{arctanh}(\bar{E})+2) \quad \text { when } \bar{E}>0,
\end{array}\right. \\
& \theta_{2}=\left\{\begin{array}{l}
\frac{1}{4}\left(\frac{4}{1+e^{-\bar{E}}}\right) \quad \text { when } \bar{E}<0, \\
\frac{1}{4}(\operatorname{arctanh}(\bar{E})+2) \text { when } \bar{E}>0,
\end{array}\right.
\end{aligned}
$$




$$
\begin{aligned}
& \theta_{3}= \begin{cases}\frac{1}{3}(\tanh (\bar{E})+1) & \text { when } \bar{E}<0, \\
\frac{1}{3}(\operatorname{arctanh}(\bar{E})+2) & \text { when } \bar{E}>0,\end{cases} \\
& \theta_{4}= \begin{cases}\frac{1}{3}\left(2 \int_{-\infty}^{\bar{E}}\left(1-\frac{1}{1+e^{-t^{2}}}\right) d t+\sigma\right) & \text { when } \bar{E}<0, \\
\frac{1}{3}(\operatorname{arctanh}(\bar{E})+2) & \text { when } \bar{E}>0,\end{cases}
\end{aligned}
$$

where $\bar{E}=10\left(\frac{E-E_{\min }}{E_{\max }-E_{\min }}\right)-5$ and $\sigma=\int_{-\infty}^{\infty}\left(1-\frac{1}{1+e^{-t^{2}}}\right) d t$.

\section{Optimization procedure}

Using (12), (13) and the formulae (6-11), and given a trial thickness $d$ and the parameters $c, k, p, q a_{i}$ and $b_{i}, i=1, \ldots, 4$, a theoretical transmittance $T^{\text {theor }}(\lambda)$ can be computed. Given a set of observations $T^{\text {meas }}\left(\lambda_{i}\right), i=1, \ldots, M$, the objective is to solve the following minimization problem:

$$
\text { Minimize } \sum_{i=1}^{M}\left[T^{\text {theor }}\left(\lambda_{i}\right)-T^{\text {meas }}\left(\lambda_{i}\right)\right]^{2} \text {. }
$$

The objective function (sum of squares) of (14) has many local minimizers, therefore the optimization procedure is not straightforward, since the application of an ordinary minimization algorithm will normally lead to a local-nonglobal solution of (14). On the other hand, performing complete local searches starting from many different initial points is computationally very expensive. This motivated us to develop an algorithm that classifies, in an economic way, promising initial points for local methods. The local minimization procedure uses as initial approximations the candidates so far generated by the classification scheme. The final estimate is the best point obtained by those local minimizations. Let us now describe this method, called FFM (functional form minimization) from now on, used for solving (14).

Assume that $0 \leq d_{\min } \leq d_{\max }, c_{\min } \leq c_{\max }, k_{\min } \leq k_{\max }, 1 \leq p_{\min } \leq p_{\max }, 0 \leq q_{\min } \leq$ $q_{\max }$, lower and upper bounds for parameters $d, c, k, p$ and $q$, respectively; $a_{i}^{0}, b_{i}^{0} \geq 0$ estimations of $a_{i}$ and $b_{i}, i=1, \ldots 4 ; \delta d>0, \bar{F}>0$, integers $S_{2} \geq S_{1}>0$, and $P \in(0,1]$ are given. Define $B$ as the four-dimensional box

$$
\left[c_{\min }, c_{\max }\right] \times\left[k_{\min }, k_{\max }\right] \times\left[p_{\min }, p_{\max }, \times\left[q_{\min }, q_{\max }\right],\right.
$$

and $G=(B, S)$ as the grid given by the $S^{4}$ points $y \in \mathbb{R}^{4}$ of the form $y=\left(c_{i}, k_{j}, p_{k}, q_{l}\right)$, where $c_{i}=c_{\min }+i\left(c_{\max }-c_{\min }\right) /(S-1), i=0, \ldots, S-1$ and so on. With some abuse of notation we will say that $x=\left(c, k, p, q, a_{1}, a_{2}, a_{3}, a_{4}, b_{1}, b_{2}, b_{3}, b_{4}\right) \in \mathbb{R}^{12}$ belongs to $G$ if $(c, k, p, q)$ belongs to $G$ and $a_{i}$ and $b_{i}$ are fixed at $a_{i}^{0}$ and $b_{i}^{0}$ for $i=1, \ldots, 4$. The algorithm below describes how to obtain estimations of $d$ and $x$. 


\section{Algorithm FFM}

\section{Step 1: Initialization}

Set $f_{\text {best }} \leftarrow+\infty$.

\section{Step 2: Coarse Grid}

For all integer $d=d_{\min }+i \delta d \in\left[d_{\min }, d_{\max }\right]$

$$
\begin{aligned}
& \bar{x}, \bar{f} \leftarrow \operatorname{Mesh}-\operatorname{Search}\left(d, G\left(B, S_{1}\right)\right), \\
& \text { if }\left(\bar{f}<f_{\text {best }}\right) \text { then } f_{\text {best }} \leftarrow \bar{f}, x_{\text {best }} \leftarrow \bar{x} \text { and } d_{\text {coarse }} \leftarrow d .
\end{aligned}
$$

Step 3: Fine Grid

For all integer $d \in\left[d_{\text {coarse }}-\delta d, d_{\text {coarse }}+\delta d\right]$

$$
\begin{aligned}
& \bar{x}, \bar{f} \leftarrow \operatorname{LocalSearch}\left(d, x_{\text {best }}, B, 100\right), \\
& \text { if }\left(\bar{f}<f_{\text {best }}\right) \text { then } f_{\text {best }} \leftarrow \bar{f}, d_{\text {best }} \leftarrow d .
\end{aligned}
$$

Step 4: Final improvement

$x_{\text {best }}, f_{\text {best }} \leftarrow$ Mesh-Search $\left(d_{\text {best }}, G\left(B, S_{2}\right)\right)$.

Subroutine Mesh-Search $(d, G(B, S))$

Step 1: Set $K$ as the set of pairs $(x, F(x))$ such that $x \in G$.

Step 2: Set $K^{\prime}=\emptyset$.

For all $(x, f) \in K$

$$
\text { if }(f<\bar{F}) \text { then } \bar{x}, \bar{f} \leftarrow \text { LocalSearch }(d, x, B, 1) \text { and } K^{\prime}=K^{\prime} \cup\{(\bar{x}, \bar{f})\} \text {. }
$$

Step 3: Set $K^{\prime \prime} \subseteq K^{\prime}$ as the subset of pairs $(x, f) \in K^{\prime}$ such that $f$ is among the $\min \left(P\left|G^{\prime}\right|, 10\right)$ smaller functional values.

Set $f_{\text {best }} \leftarrow+\infty$.

For all $(x, f) \in K^{\prime \prime}$

$$
\begin{aligned}
& \bar{x}, \bar{f} \leftarrow \text { LocalSearch }(d, x, B, 100), \\
& \text { if }\left(\bar{f}<f_{\text {best }}\right) \text { then } f_{\text {best }} \leftarrow \bar{f}, x_{\text {best }} \leftarrow \bar{x} .
\end{aligned}
$$

Return $x_{\text {best }}$ and $f_{\text {best }}$.

In the LocalSearch procedure with parameters $d_{\text {fixed }}, x_{0}, B$ and maxit we use the software BOX-QUACAN (see $[4,5]$ ) to find a local minimizer of the objective function of (14) restricted to the box $B$. The thickness is fixed at $d_{\text {fixed }}$ and the method starts from $x_{0}$. The maximum number of allowed iterations is maxit. Although $a_{i}$ and $b_{i}$ are not bounded, we restricted each of them to the boxes $\left[a_{\min }, a_{\max }\right]$ and $\left[b_{\min }, b_{\max }\right]$. 


\section{Computational results}

The method described in the previous section has been used for estimating optical constants of several computer-generated and real films. We used all the films considered in [1] and [6], as well as some very thin computer-generated films.

In the numerical tests we use $c_{\min }=k_{\min }=-10, c_{\max }=k_{\max }=10, p_{\min }=1.1$, $p_{\max }=5, q_{\min }=0, q_{\max }=0.5, a_{\min }=b_{\min }=0, a_{\max }=b_{\max }=10, a_{i}^{0}=0.25, b_{i}^{0}=1$, $i=1, \ldots 4, \bar{F}=10, S_{2}=5, S_{1}=3$, and $P=0.1$. Values of $d_{\min }, d_{\max }$ and $\delta d$ are different for each film and depend on a previous acknowledge of a range to which the true thickness belongs. We use $d_{\min }=10, d_{\max }=200$ and $\delta d=10$ for the computer-generated films $\mathrm{A}$, $\mathrm{C}$ and $\mathrm{E}$ in [1], $d_{\min }=300, d_{\max }=900$ and $\delta d=10$ for the computer-generated films $\mathrm{B}$ and $\mathrm{D}$ in [1], and $d_{\min }=50, d_{\max }=1300$ and $\delta d=50$ for the real films in [6].

All the experiments were run on a Intel Pentium III Computer with the following main characteristics: $728 \mathrm{Mbytes}$ of RAM, 1GHz. Codes are in Fortran77 and the compiler used was GNU Fortran 0.5.25, with the optimization option "-O3".

\subsection{Computer-generated and real films in [1] and [6]}

The films in [1] are called A, B, C, D and E. Table 1 reports the true thickness and the thickness obtained by both PUMA and FFM, as well as their quadratic errors. Figures 1-5 show the real and estimated values for the refractive index and the absorption coefficient obtained by the two methods. Table 2 shows the estimated thickness and the quadratic errors obtained by FFM and PUMA in the real films of [6]. This real films correspond to a series of six a-Si:H (hidrogenated amorphous silicon) samples grown under identical conditions, but with thickness varying, approximately, from 100 to 1200nm. Figure 6 shows the estimated values retrieved for this films.

\subsection{Estimation of very thin films}

The computer-generated film E, described in [1], has the refractive index and the absorption coefficient displayed in Figure 7. In [1] and in the previous subsection the thickness of that film was $80 \mathrm{~nm}$. This is the thinnest thickness for which we could obtain reasonable results using PUMA. Here we generated transmittances for films with the same characteristics but decreasing values of thickness. Namely, we used the thicknesses: 70, 60, 50, 40, 30, 20, and 10nm. Figure 8 shows the generated transmittances for each of these thicknesses. Table 3 shows the estimated thickness obtained by PUMA and FFM for that films. Figures 9 and 10 show the estimated values for the refractive index and the absorption coefficient obtained by both methods.

\section{$5 \quad$ Final remarks}

We introduced a new method for the estimation of parameters of thin films. It is based on a flexible functional definition for the absorption curve, which allows one to provide more a priori information to an optimization procedure, than the one that is provided by pointwise optimization schemes $[1,2,3,6]$. However, the number of parameters and the flexibility of the model is enough to guarantee good fitness of the transmittance curves. 
The method behaved very well with many films for which the pointwise approach had been successful and, moreover, was also very efficient to recover the parameters of very thin computer-generated films.

\section{References}

[1] E. G. Birgin, I. Chambouleyron and J. M. Martínez, Estimation of the optical constants and the thickness of thin films using unconstrained optimization, Journal of Computational Physics 151, pp. 862-880 (1999).

[2] I. E. Chambouleyron, J. M. Martínez, A. C. Moretti and M. Mulato, Optical constants of thin films by means of a pointwise constrained optimization approach, Thin Solid Films 317, pp. 133-136 (1998).

[3] I. E. Chambouleyron, J. M. Martínez, A. C. Moretti and M. Mulato, The retrieval of the optical constants and the thickness of thin films from transmission spectra, Applied Optics 36, pp. 8238-8247 (1997).

[4] A. Friedlander, J. M. Martínez and S. A. Santos, A new trust region algorithm for bound constrained minimization. Applied Mathematics and Optimization 30, pp. 235-266 (1994).

[5] www.ime.unicamp.br/ martinez

[6] M. Mulato, I. Chambouleyron, E. G. Birgin and J. M. Martínez, Determination of thickness and optical constants of amorphous silicon films from transmittance data, Applied Physics Letters 77, pp. 2133-2135 (2000).

[7] M. Raydan, The Barzilai and Borwein gradient method for the large unconstrained minimization problem, SIAM Journal on Optimization 7, pp. 26-33 (1997).

[8] R. Swanepoel, Determination of the thickness and optical constants of amorphous silicon, J. Phys. E: Sci. Instrum. 16, pp. 1214-1222 (1983).

[9] S. H. Wemple, Refractive-index behavior of amorphous semiconductors and glasses, Phys. Rev. B 7, pp. 3767-3777 (1973). 


\section{Tables}

Table 1: Retrieved thicknesses and quadratic errors obtained by FFM and PUMA for the computer-generated films in [1].

\begin{tabular}{c|c|rr|rr}
\hline & & \multicolumn{2}{|c}{ FFM } & \multicolumn{2}{c}{ PUMA } \\
\hline Film & $d^{\text {true }}$ & $d^{\text {retr }}$ & Quadratic error & $d^{\text {retr }}$ & Quadratic error \\
\hline A & 100 & 100 & $1.07 \times 10^{-5}$ & 100 & $6.33 \times 10^{-6}$ \\
B & 600 & 601 & $1.37 \times 10^{-4}$ & 600 & $2.42 \times 10^{-5}$ \\
C & 100 & 109 & $1.47 \times 10^{-8}$ & 100 & $6.09 \times 10^{-8}$ \\
D & 600 & 600 & $1.28 \times 10^{-7}$ & 600 & $6.35 \times 10^{-8}$ \\
E & 80 & 80 & $1.70 \times 10^{-7}$ & 80 & $5.08 \times 10^{-7}$ \\
\hline
\end{tabular}

Table 2: Retrieved thicknesses and quadratic errors obtained by FFM and PUMA for the real films in $[6]$.

\begin{tabular}{rr|rr}
\hline \multicolumn{2}{c}{ FFM } & \multicolumn{2}{c}{ PUMA } \\
\hline$d^{\text {retr }}$ & Quadratic error & $d^{\text {retr }}$ & Quadratic error \\
\hline 99 & $3.09 \times 10^{-3}$ & 98 & $1.61 \times 10^{-3}$ \\
162 & $2.85 \times 10^{-3}$ & 163 & $3.97 \times 10^{-4}$ \\
236 & $3.25 \times 10^{-3}$ & 234 & $9.58 \times 10^{-4}$ \\
311 & $5.34 \times 10^{-3}$ & 310 & $1.18 \times 10^{-3}$ \\
628 & $9.62 \times 10^{-3}$ & 624 & $1.60 \times 10^{-3}$ \\
1225 & $1.56 \times 10^{-2}$ & 1220 & $1.40 \times 10^{-3}$ \\
\hline
\end{tabular}

Table 3: Retrieved thicknesses and quadratic errors obtained by FFM and PUMA for the very thin computer-generated films (thicknesses ranging from 10nm up to $70 \mathrm{~nm}$ ).

\begin{tabular}{c|rr|rr}
\hline & \multicolumn{2}{|c}{ FFM } & \multicolumn{2}{c}{ PUMA } \\
\hline$d^{\text {true }}$ & $d^{\text {retr }}$ & Quadratic error & $d^{\text {retr }}$ & Quadratic error \\
\hline 10 & 61 & $2.04 \times 10^{-5}$ & 2 & $1.52 \times 10^{-7}$ \\
20 & 143 & $2.92 \times 10^{-5}$ & 56 & $1.04 \times 10^{-5}$ \\
30 & 30 & $1.42 \times 10^{-6}$ & 51 & $1.69 \times 10^{-5}$ \\
40 & 38 & $1.52 \times 10^{-6}$ & 60 & $5.42 \times 10^{-8}$ \\
50 & 50 & $7.57 \times 10^{-7}$ & 59 & $1.60 \times 10^{-5}$ \\
60 & 60 & $1.28 \times 10^{-7}$ & 60 & $2.85 \times 10^{-5}$ \\
70 & 70 & $5.56 \times 10^{-7}$ & 78 & $6.13 \times 10^{-7}$ \\
\hline
\end{tabular}




\section{$7 \quad$ Figure captions}

Figure 1: "True" (dashed lines) and retrieved values (open circles by FFM and open triangles by PUMA) for the transmittance, the refractive index, and the absorption coefficient of numerically-generated Film A of [1].

Figure 2: "True" (dashed lines) and retrieved values (open circles by FFM and open triangles by PUMA) for the transmittance, the refractive index, and the absorption coefficient of numerically-generated Film B of [1].

Figure 3: "True" (dashed lines) and retrieved values (open circles by FFM and open triangles by PUMA) for the transmittance, the refractive index, and the absorption coefficient of numerically-generated Film $\mathrm{C}$ of [1].

Figure 4: "True" (dashed lines) and retrieved values (open circles by FFM and open triangles by PUMA) for the transmittance, the refractive index, and the absorption coefficient of numerically-generated Film D of [1].

Figure 5: "True" (dashed lines) and retrieved values (open circles by FFM and open triangles by PUMA) for the transmittance, the refractive index, and the absorption coefficient of numerically-generated Film E of [1].

Figure 6: Retrieved values for the refractive index and the absorption coefficient of the real film in [6]. Note the unrealistic large values found with FFM for the refractive index at small wavelengths. This is a limitation of the model chosen to represent $n$ [9] which is not applicable to photon energies close to the single-effective-oscillator energy model.

Figure 7: "True" values for the refractive index (left side) and the absorption coefficient (right side) of the numerically-generated Film E.

Figure 8: Transmittances of very thin films with thicknesses ranging from 10nm up to $70 \mathrm{~nm}$ and the same refractive index and absorption coefficient of the numericallygenerated Film E.

Figure 9: "True" (dashed lines) and retrieved values (open circles by FFM and open triangles by PUMA) for the refractive index of numerically-generated very thin films.

Figure 10: "True" (dashed lines) and retrieved values (open circles by FFM and open triangles by PUMA) for the absorption coefficient of numerically-generated very thin films. 

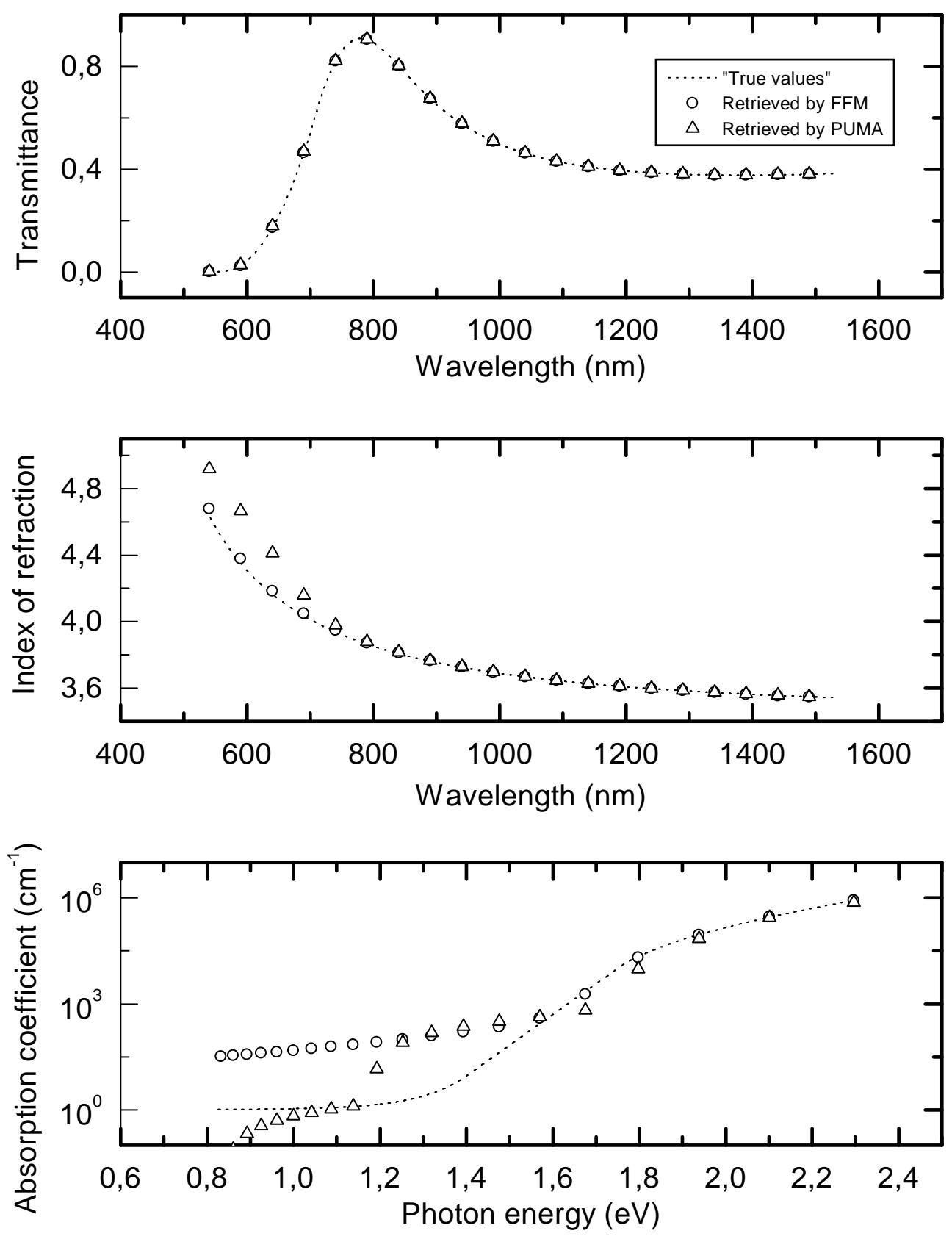

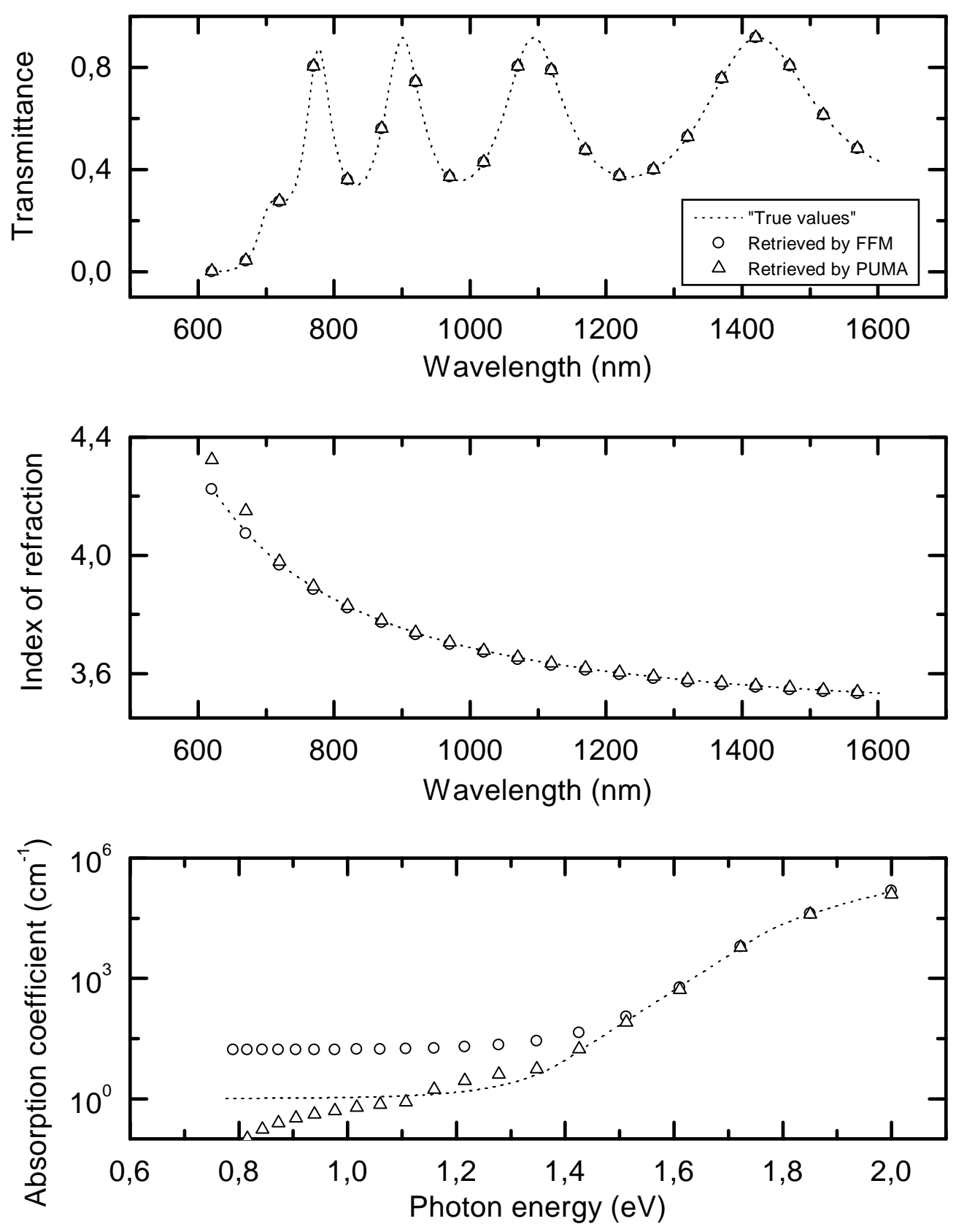

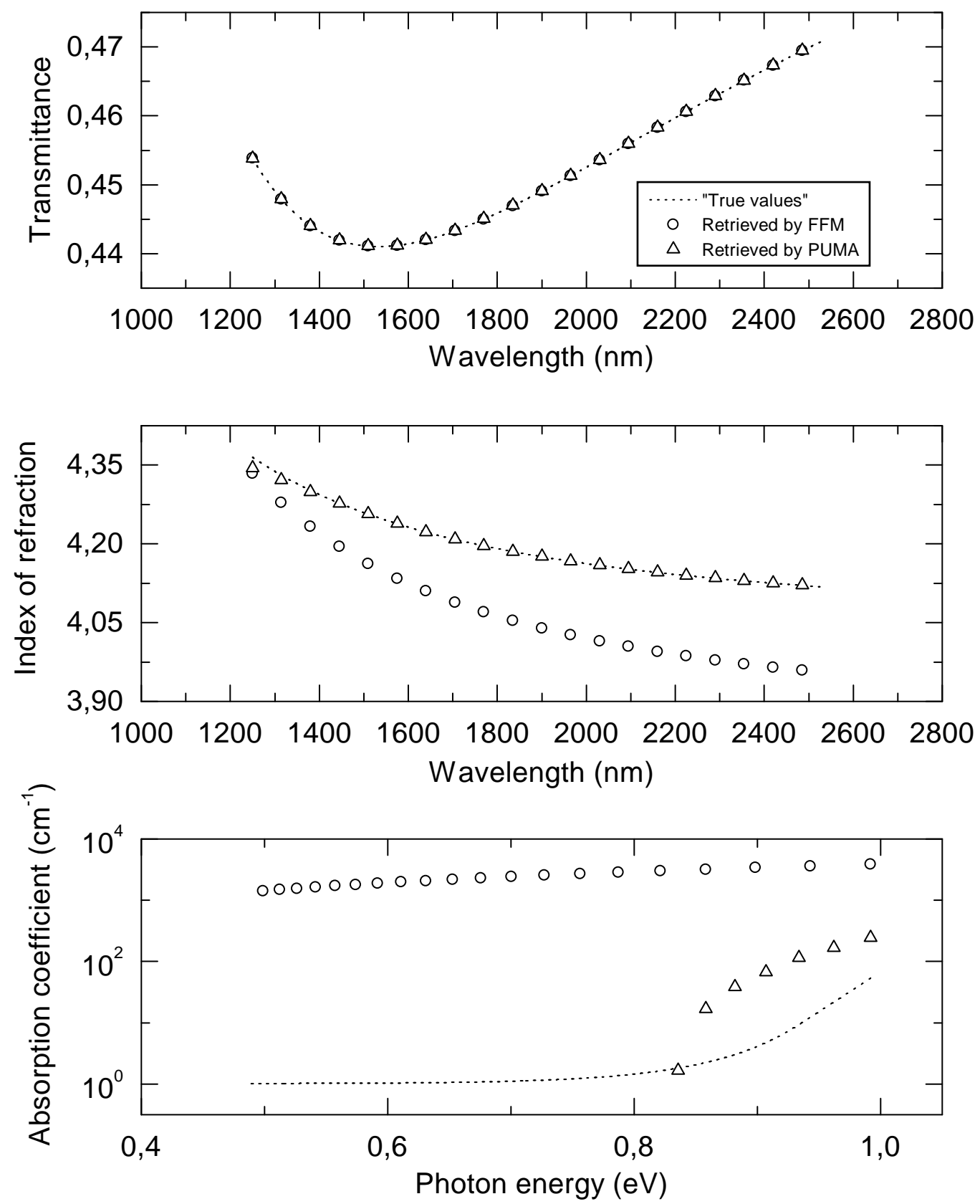

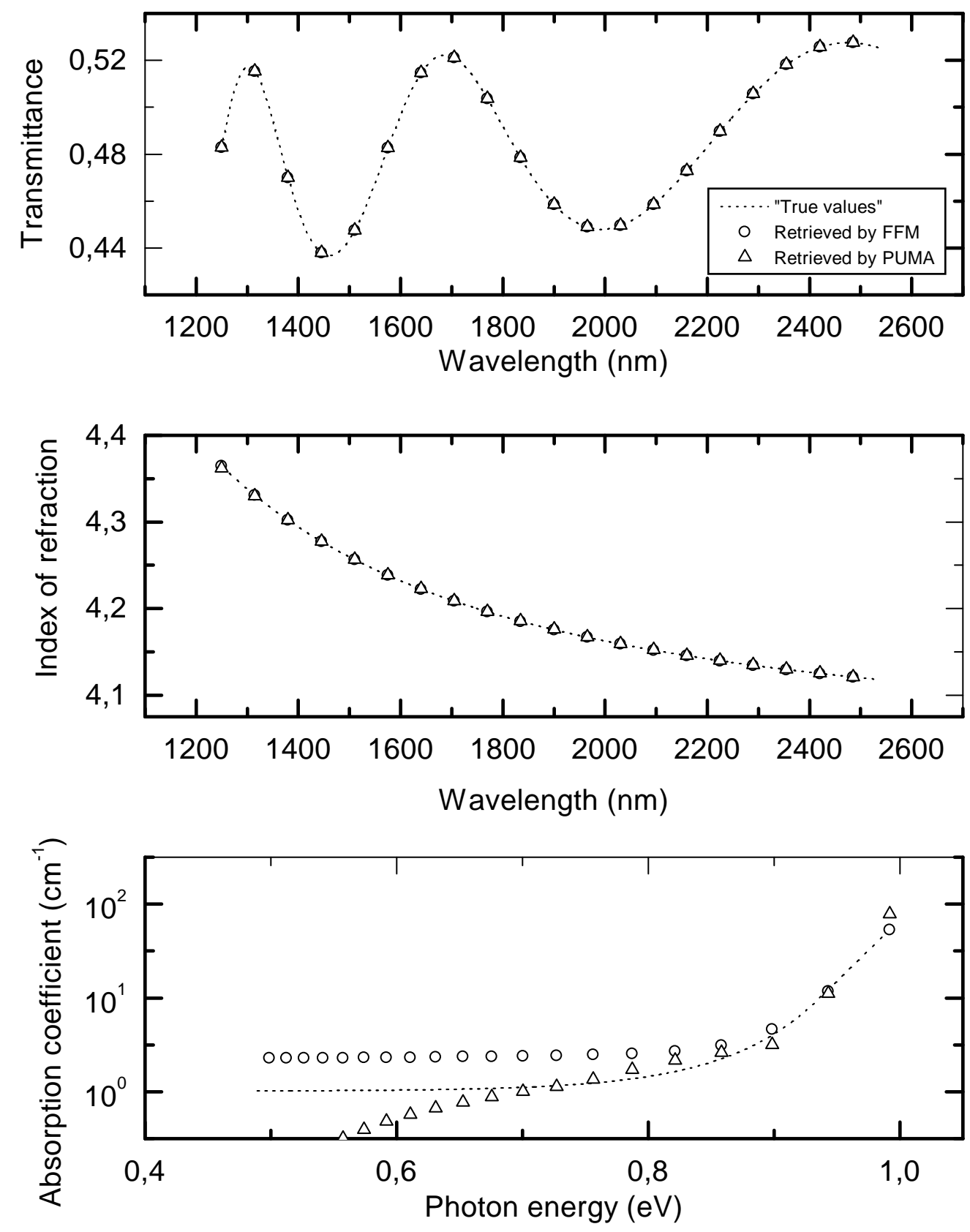

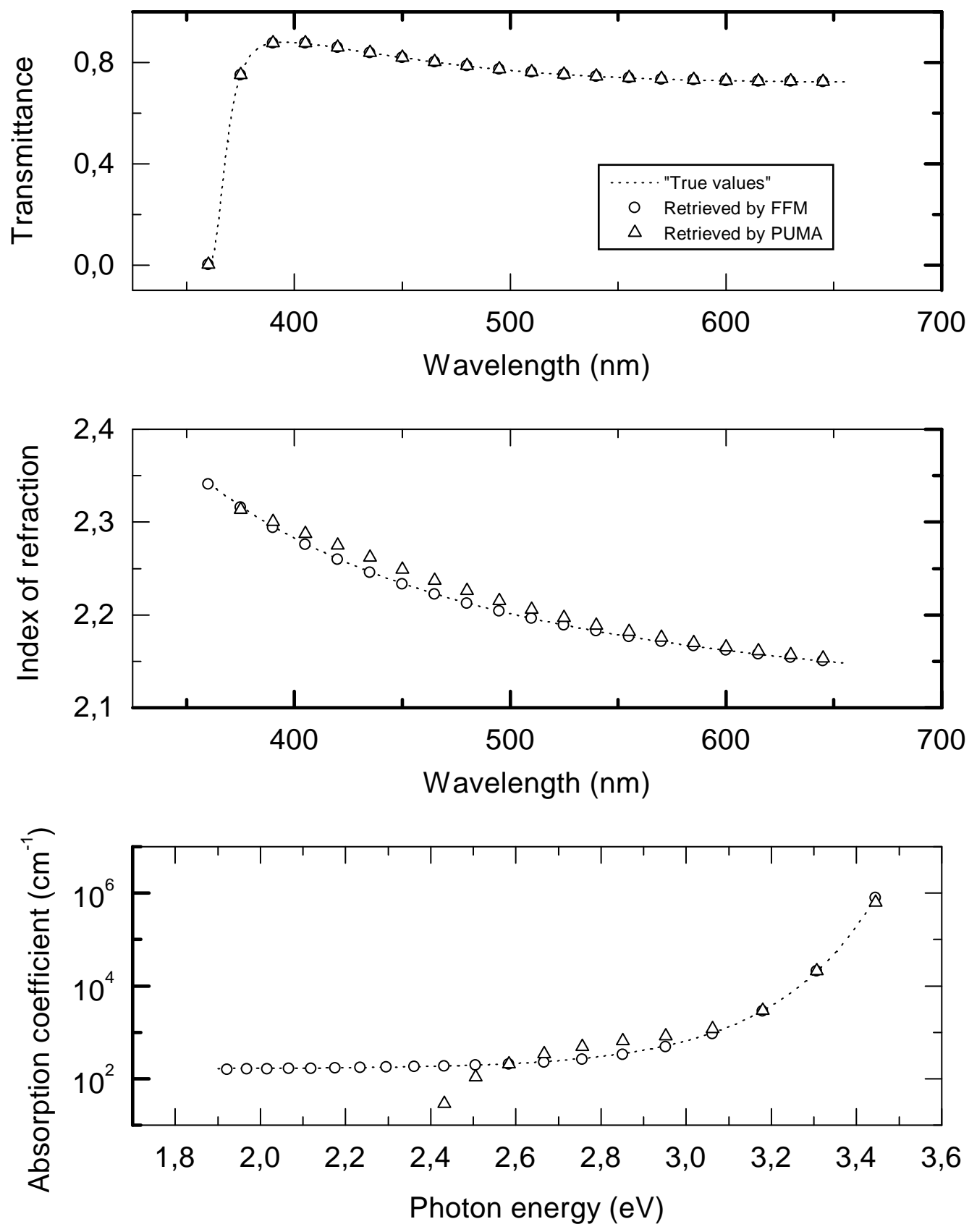

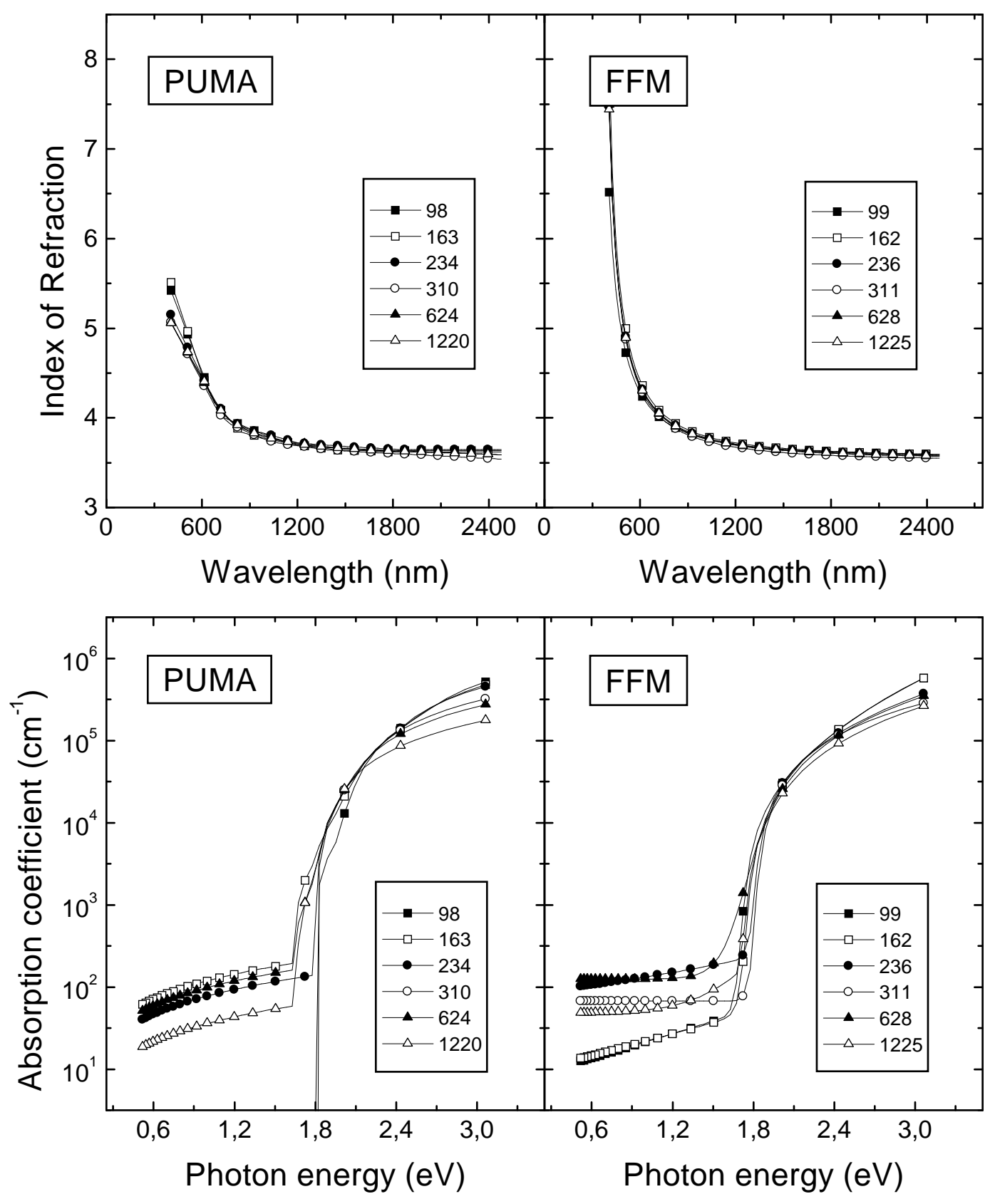

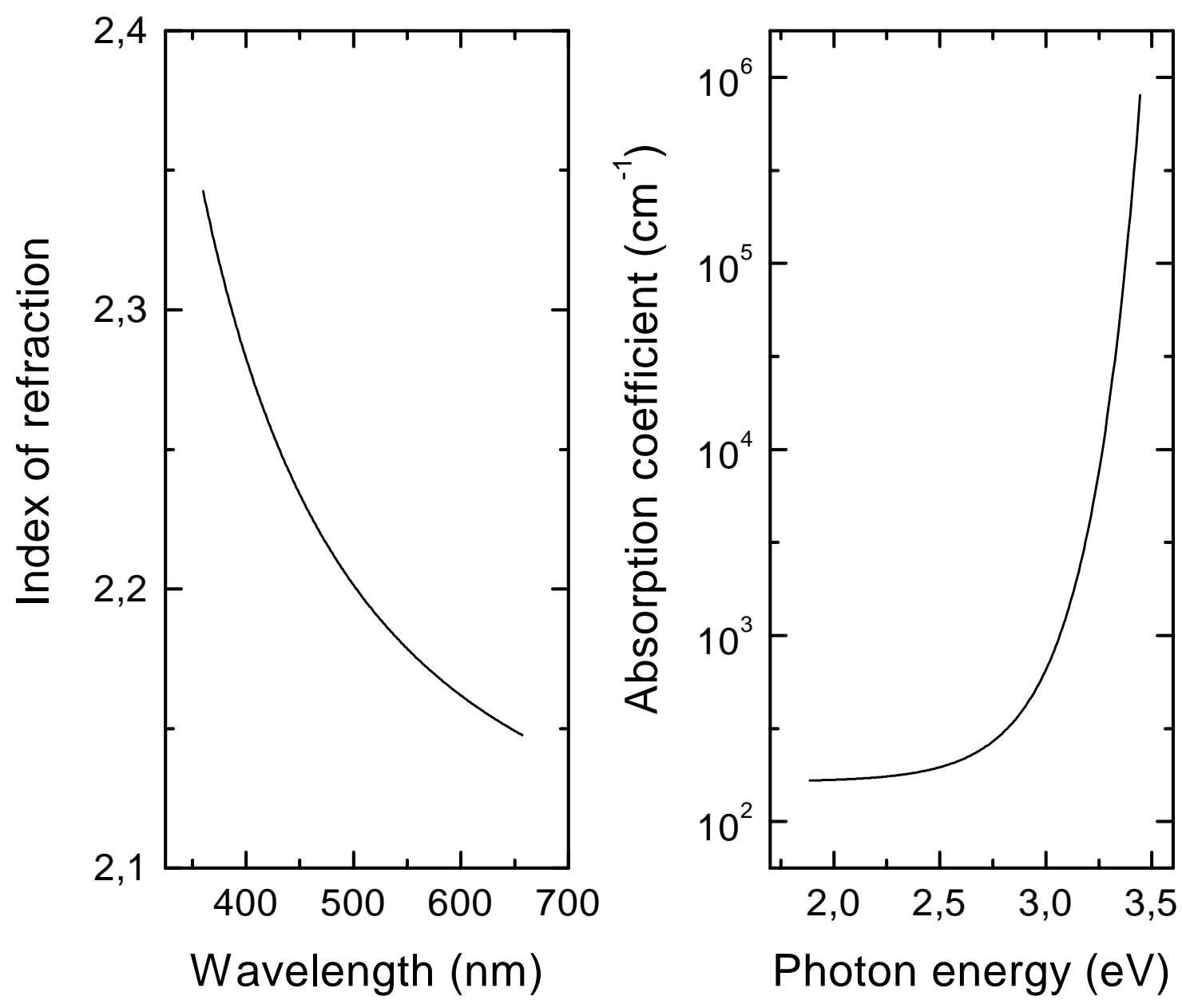


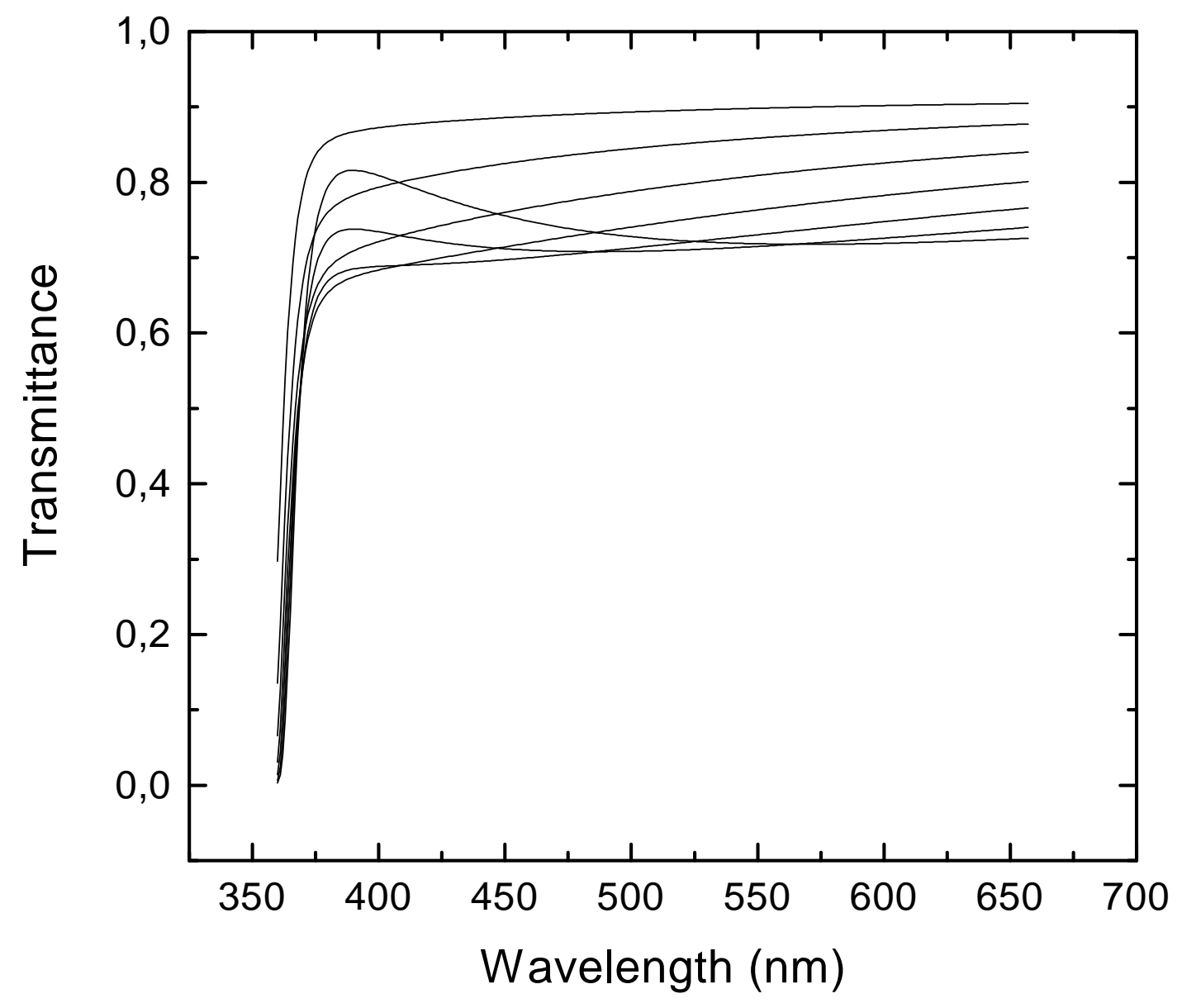




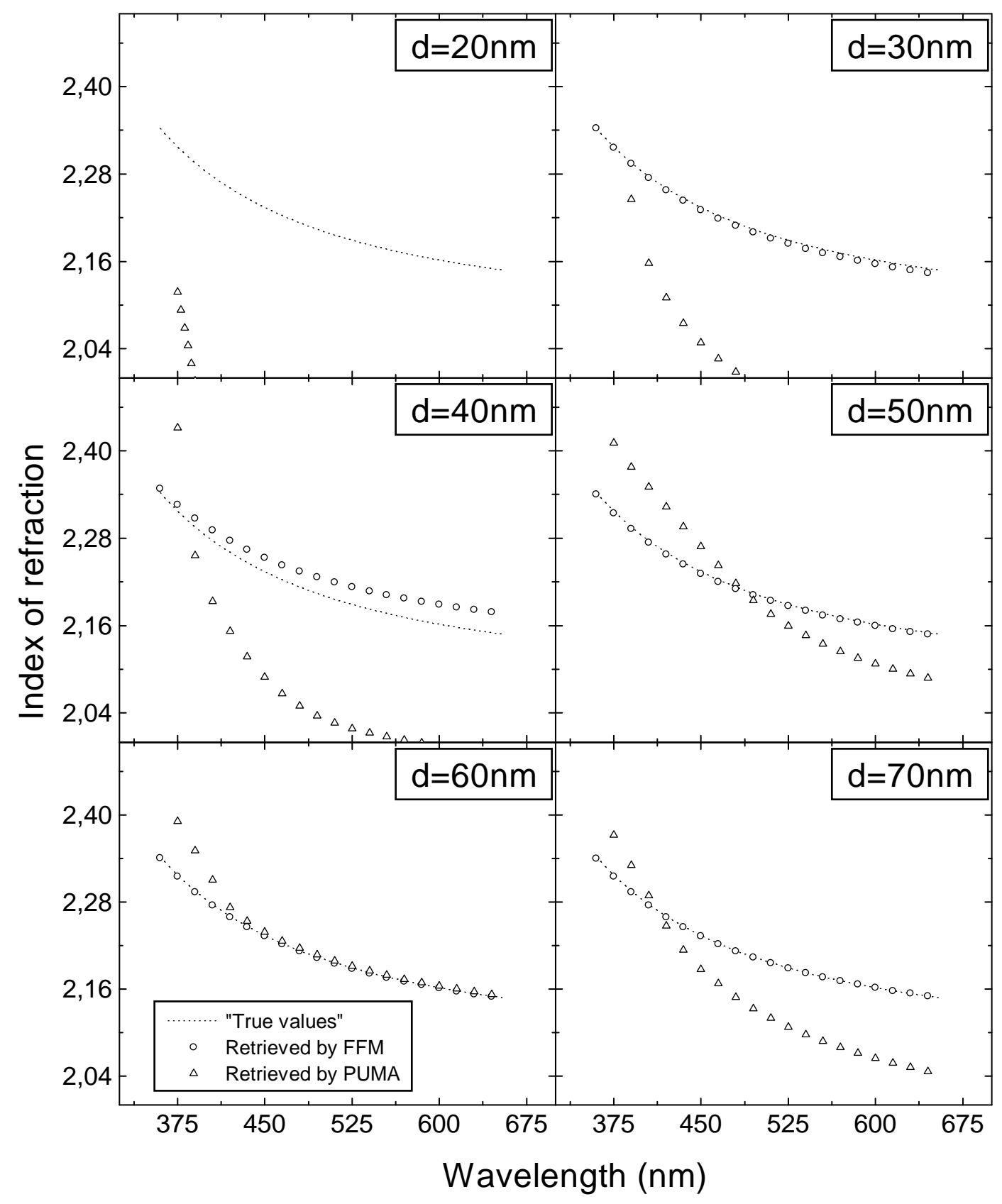




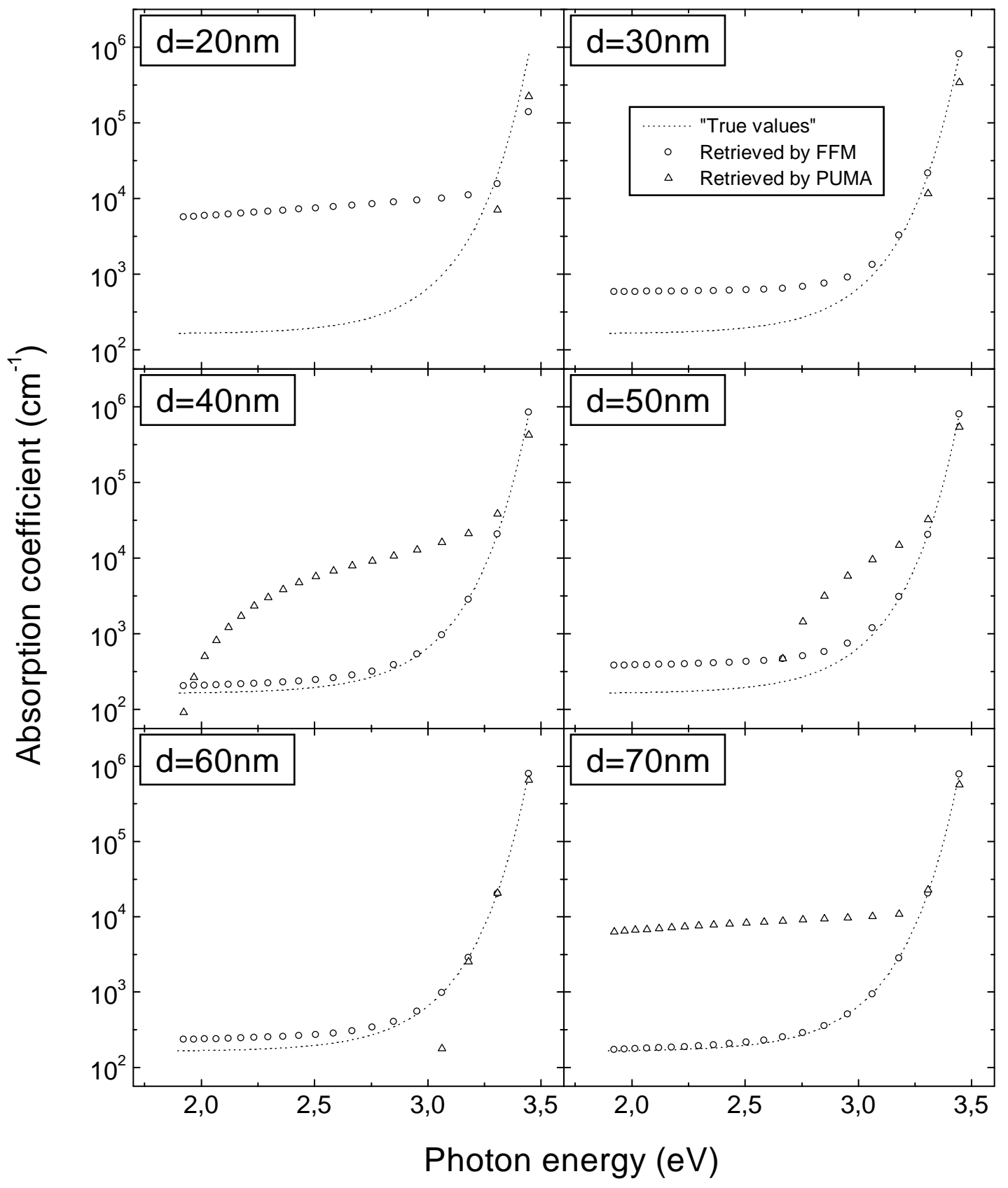

